\title{
The dusty aftermath of SN Hunt 248: merger-burst remnant?
}

\author{
Jon C. Mauerhan, ${ }^{1 \star}$ Schuyler D. Van Dyk, ${ }^{2}$ Joel Johansson, ${ }^{3}$ Ori D. Fox, ${ }^{4}$ \\ Alexei V. Filippenko ${ }^{1,5}$ and Melissa L. Graham ${ }^{1,6}$ \\ ${ }^{1}$ Department of Astronomy, University of California, Berkeley, CA 94720-3411, USA \\ ${ }^{2}$ Caltech/IPAC, 100-22, Pasadena, CA 91125, USA \\ ${ }^{3}$ Department of Particle Physics and Astrophysics, Weizmann Institute of Science, 234 Herzl St, Rehovot, Israel \\ ${ }^{4}$ Space Telescope Science Institute, 3700 San Martin Drive, Baltimore, MD 21218, USA \\ ${ }^{5}$ Senior Miller Fellow, Miller Institute for Basic Research in Science, University of California, Berkeley, CA 94720, USA \\ ${ }^{6}$ Department of Astronomy, University of Washington, Box 351580, Seattle, WA 98195-1580, USA
}

Accepted 2017 September 25. Received 2017 August 4; in original form 2017 January 31

\begin{abstract}
SN Hunt 248 was classified as a non-terminal eruption (a supernova 'impostor') from a directly identified and highly variable cool hypergiant star. The 2014 outburst achieved peak luminosity equivalent to that of the historic eruption of luminous blue variable (LBV) $\eta$ Car, and exhibited a multipeaked optical light curve which rapidly faded after $\sim 100 \mathrm{~d}$. We report ultraviolet (UV) through optical observations of SN Hunt 248 with the Hubble Space Telescope (HST) about $1 \mathrm{yr}$ after the outburst, and mid-infrared observations with the Spitzer Space Telescope before the burst and in decline. The HST data reveal a source which is a factor of $\sim 10$ dimmer in apparent brightness than the faintest available measurement of the precursor star. The UVoptical spectral energy distribution (SED) requires a strong Balmer continuum, consistent with a hot B4-B5 photosphere attenuated by grey circumstellar extinction. Substantial mid-infrared excess of the source is consistent with thermal emission from hot dust with a mass of $\sim 10^{-6}$ $10^{-5} \mathrm{M} \odot$ and a geometric extent which is comparable to the expansion radius of the ejecta from the 2014 event. SED modelling indicates that the dust consists of relatively large grains $(>0.3 \mu \mathrm{m})$, which could be related to the grey circumstellar extinction which we infer for the UV-optical counterpart. Revised analysis of the precursor photometry is also consistent with grey extinction by circumstellar dust, and suggests that the initial mass of the star could be twice as large as previously estimated (nearly $\sim 60 \mathrm{M}_{\odot}$ ). Re-analysis of the earlier outburst data shows that the peak luminosity and outflow velocity of the eruption are consistent with a trend exhibited by stellar merger candidates, prompting speculation that SN Hunt 248 may also have stemmed from a massive stellar merger or common-envelope ejection.
\end{abstract}

Key words: stars: evolution-supernovae: general - supernovae: individual: SN Hunt 248 stars: winds, outflows.

\section{INTRODUCTION}

'Supernova impostors' are a heterogeneous class of transient exhibiting luminosities between those of classical novae and supernovae ( $\mathrm{SNe}$ ), and a wide variety of light curves and spectral features (Smith et al. 2011; Van Dyk \& Matheson 2012a). Some have been broadly characterized as extragalactic analogues to the historic super-Eddington eruptions of the Galactic luminous blue variable (LBV) stars $\eta$ Carinae and P Cygni (Humphreys \& Davidson 1994; Van Dyk 2000; Smith et al. 2011, 2016a; Humphreys et al. 2016). However, the physical mechanisms involved remain unclear. Indeed, the variety of transients classifiable as $\mathrm{SN}$ impostors suggests that there are multiple evolutionary channels. Current possibilities include instabilities associated with late-stage nuclear burning (Shiode \& Quataert 2014; Smith \& Arnett 2014), violent binary encounters (Soker 2004; Kashi \& Soker 2010; Smith \& Frew 2011) and stellar mergers involving massive binary star systems (Soker \& Kashi 2013; Kochanek 2014; Smith et al. 2016b).

Recent studies of the fading optical-infrared (IR) remnants of luminous transients have shown that objects previously classified as SN impostors might actually be terminal explosions after all, in which a stellar core collapses, but with an incomplete or failed expulsion of the stellar mantle. Indeed, the fate of the prototype impostor SN 1997bs (Van Dyk et al. 2000) has recently come under question, based on the unexpectedly low luminosity for the optical-IR remnant relative to the directly identified stellar precursor (Adams $\&$ Kochanek 2015). The fate of other historic transients for which 

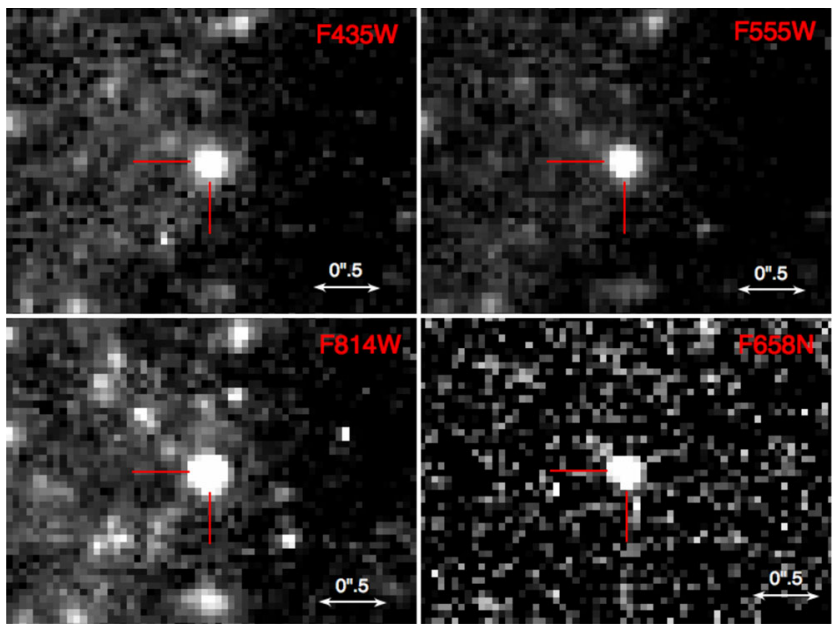

Figure 1. HST/WFPC2 and ACS images (log stretch) of the cool hypergiant precursor of SN Hunt 248 (reproduced from data presented by Mauerhan et al. 2015), from $3374 \mathrm{~d}$ before the onset of the 2014 eruption (broad-band filter images; the $F 658 \mathrm{~N}$ image is from $3715 \mathrm{~d}$ before eruption). North is up and east is towards the left.

high-quality precursor data were not available has also been the source of ongoing debate (e.g. SN 1961V; Kochanek, Szczygiel \& Stanek 2011; Smith et al. 2011; Van Dyk \& Matheson 2012b), in part because the cooling outflows from nonterminal eruptions can form dust that obscures the star, and also because late-time line emission can result from persistent interaction between the outflow and an extended distribution of slower pre-existing circumstellar material (CSM). These issues underscore the importance of obtaining latetime multiwavelength monitoring observations of SN impostors, in order to track their post-outburst evolution and determine their ultimate fate.

SN Hunt 248 was a luminous transient in NGC 5806 classified as an $\mathrm{SN}$ impostor. The light curve exhibited a main peak equivalent in luminosity to the peak of $\eta$ Car's historic outburst in the 1840 s, and another subsequent peak of longer duration which was likely the result of interaction between the erupted material and slower pre-existing CSM expelled prior to the outburst (Kankare et al. 2015; Mauerhan et al. 2015). A particularly interesting aspect of SN Hunt 248 is the detection of the luminous precursor star in archival data, shown in Fig. 1 (images reproduced from Mauerhan et al. 2015). Multicolour photometry from the Hubble Space Telescope (HST) showed that the stellar precursor's position on the Hertzsprung-Russell (HR) diagram was consistent with that of a cool hypergiant star. The subsequent giant eruption from the star in 2014 provided observational support for a hypothesis that cool hypergiants might actually be relatively hot LBV stars enshrouded in an opaque wind that creates an extended pseudophotosphere (Smith, Vink \& de Koter 2004). Detailed study of the aftermath of the eruption thus provides an interesting opportunity to probe the post-outburst state and recovery of the stellar remnant.

Here, we present ultraviolet (UV) through IR observations of SN Hunt 248 with the HST and the Spitzer Space Telescope about $1 \mathrm{yr}$ after the giant outburst. In Section 3, we model the mid-IR data as a source of thermal dust emission. In Section 4, we discuss the effects of circumstellar extinction and implications for the nature of the remnant star. The times of all observation epochs are presented as days past $V$-band peak on 2014 June 21 (MJD 56830.3; UT dates are used throughout this paper). A foreground interstellar extinction value of $A_{V}=0.14 \mathrm{mag}$ has been adopted (Mauerhan et al. 2015).
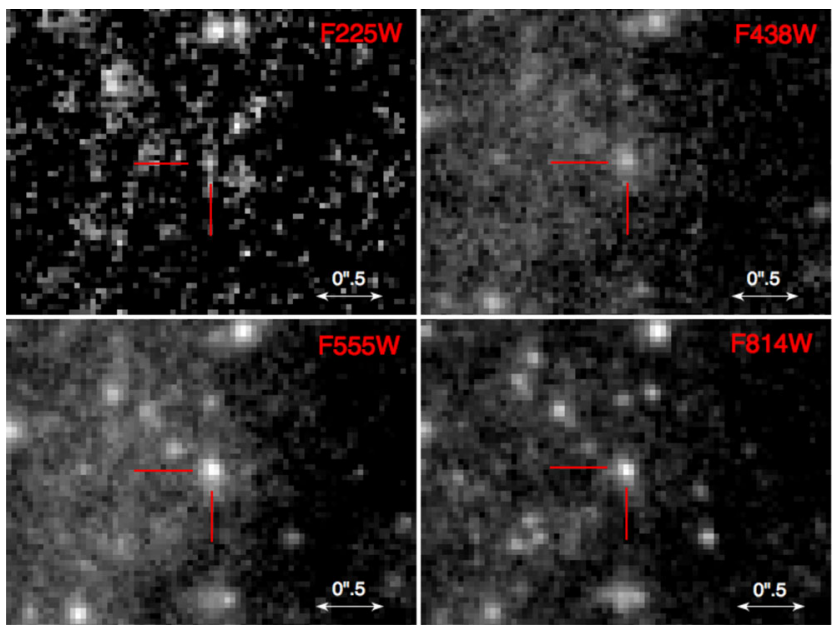

Figure 2. HST/WFC3 images (log stretch) of the remnant of SN Hunt 248 , $\sim 1$ yr after the peak of the 2014 eruption. North is up and east is towards the left.

Table 1. HST photometry of the remnant of SN Hunt 248.

\begin{tabular}{lcccc}
\hline Instrument/band & Magnitude & Flux $(\mu J y)$ & MJD & Epoch $(\mathrm{d})$ \\
\hline WFC3/F225W & $25.16 \pm 0.09$ & $0.068 \pm 0.006$ & 57204.05 & 374 \\
WFC3/F438W & $25.84 \pm 0.05$ & $0.193 \pm 0.010$ & 57204.00 & 374 \\
WFC3/F555W & $25.46 \pm 0.03$ & $0.243 \pm 0.007$ & 57199.87 & 370 \\
ACS/F814W & $24.51 \pm 0.04$ & $0.386 \pm 0.015$ & 57200.38 & 371 \\
\hline
\end{tabular}

Notes. Uncertainties are statistical. Epochs are given as days from $V$-band peak (MJD 56830.3; Mauerhan et al. 2015).

\section{OBSERVATIONS}

\subsection{HST imaging}

High-resolution imaging observations of SNHunt 248 were performed with the HST Wide-field Camera 3 (HST/WFC3) on 2015 June 26 and 30 (369 and $374 \mathrm{~d}$ after the peak of the 2014 eruption) under HST programmes GO-13684 and GO-13822 (PIs S. Van Dyk and G. Folatelli, respectively). Exposures were obtained in the $F 225 W(\mathrm{NUV}), F 438 W(B), F 555 W(V)$ and $F 814 W(I)$ filters. A point source at the position of SN Hunt 248 is securely detected in all bands, as shown in Fig. 2. Photometry of the source was extracted from the images using DOLPHOT (Dolphin 2000). We tried two different approaches to estimate the background, including the use of an annulus region to measure the sky (FitSky=1) and, alternatively, measuring the sky within the point spread function (PSF) aperture (FitSky $=3$, best to use when the field is very crowded). Our annulus-based background subtraction produced the most consistent results for all bands, although the results from each setting are within the respective uncertainties. The photometry is listed in Table 1.

\subsection{Spitzer imaging}

SN Hunt 248 was observed on five epochs during the Spitzer Space Telescope Warm Mission utilizing channels $1(3.6 \mu \mathrm{m})$ and 2 $(4.5 \mu \mathrm{m})$ of the Infrared Array Camera (IRAC; Fazio et al. 2004). We acquired fully co-added and calibrated data from the Spitzer Heritage Archive $^{1}$ from programme IDs 61063 (PI K. Sheth), 10152

\footnotetext{
${ }^{1}$ http://sha.ipac.caltech.edu/applications/Spitzer/SHA/
} 

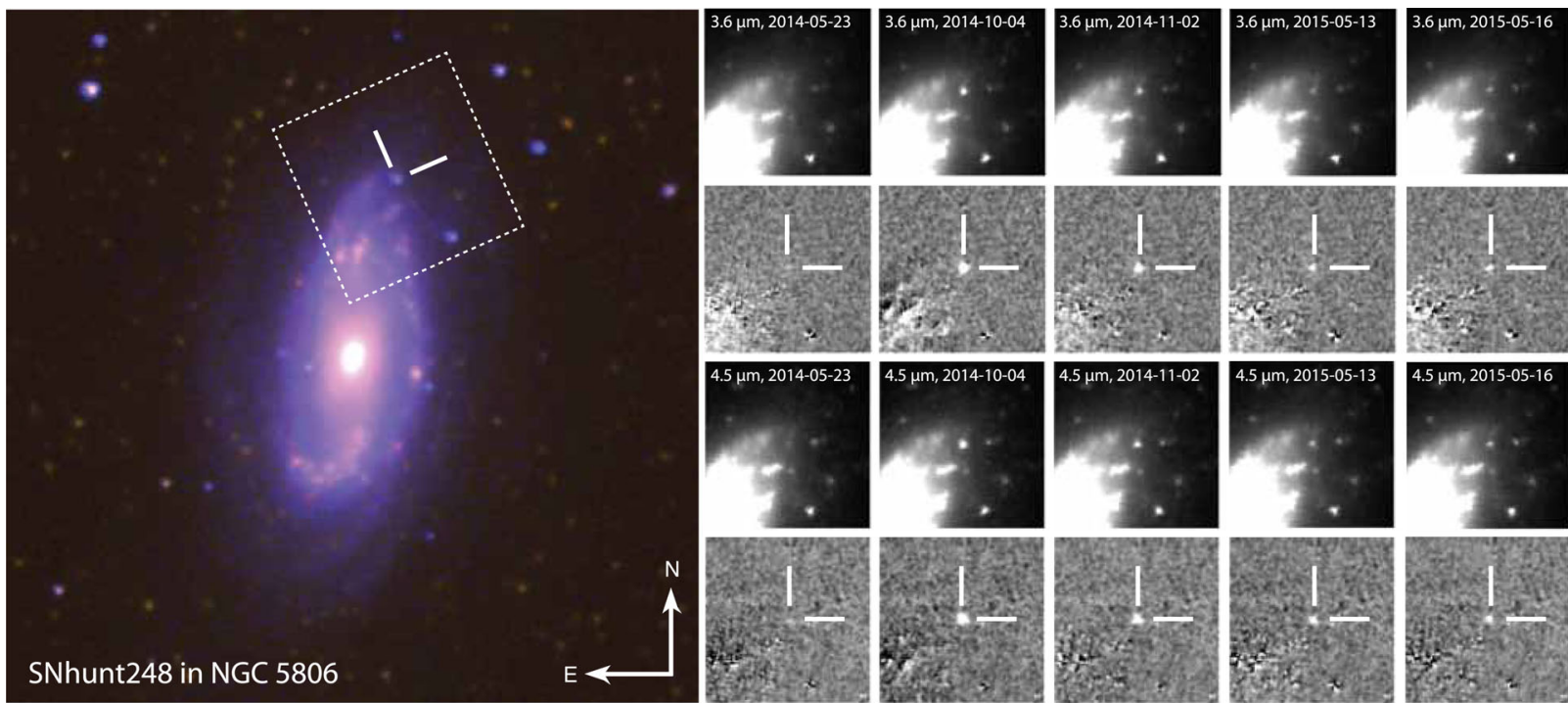

Figure 3. Colour composite of the $3.6 \mu \mathrm{m}$ (green) and $4.5 \mu \mathrm{m}$ (red) Spitzer/IRAC template images of NGC 5806 and a Palomar Transient Factory $R$-band image (blue), and the template-subtracted images of the region around SN Hunt 248 (tiled frames).

Table 2. Spitzer/IRAC photometry of SN Hunt 248.

\begin{tabular}{lcccc}
\hline MJD & Epoch (d) & $3.6 \mu \mathrm{m}$ & $4.5 \mu \mathrm{m}$ & Programme ID (PI) \\
\hline 55066.9 & -1763 & $<9.49$ & $<5.99$ & 61063 (Sheth) \\
56800.7 & -30 & $19.35 \pm 6.11$ & $11.81 \pm 5.09$ & 10152 (Kasliwal) \\
56934.6 & 104 & $110.26 \pm 6.38$ & $96.41 \pm 5.86$ & 10152 (Kasliwal) \\
56963.4 & 133 & $65.56 \pm 6.14$ & $59.70 \pm 5.91$ & 10139 (Fox) \\
57155.5 & 325 & $32.72 \pm 7.82$ & $29.26 \pm 4.76$ & 11053 (Fox) \\
57158.2 & 328 & $34.30 \pm 5.44$ & $29.57 \pm 5.61$ & 11053 (Fox) \\
\hline
\end{tabular}

Notes. Fluxes are in units of $\mu \mathrm{Jy}$. Uncertainties are statistical. Epochs are given as days from $V$-band peak (MJD 56830.3; Mauerhan et al. 2015).

(PI M. Kasliwal) and 11053 (PI O. Fox). The images for all epochs were registered with an earlier pre-outburst image of the host galaxy, which was used as a subtraction template. The template-subtracted images are shown in Fig. 3. We performed aperture photometry on the template-subtracted (PBCD / Level 2) images using a six-pixel aperture radius and aperture corrections listed in table 4.7 of the Spitzer IRAC Instrument Handbook. ${ }^{2}$ The infrared photometry are listed in Table 2.

\section{RESULTS AND ANALYSIS}

The absolute-magnitude light curve of SNHunt 248 is shown in Fig. 4, including data from Mauerhan et al. (2015) and Kankare et al. (2015). At $\sim 1 \mathrm{yr}$ after the peak of the 2014 outburst, the source has dropped to a brightness of $V=25.46 \pm 0.03 \mathrm{mag}$, which is a factor of $\sim 10$ fainter in the optical than the faintest pre-outburst state ever measured for the stellar precursor in the year 2005 ( $V=22.91 \pm 0.01 \mathrm{mag}$; see Mauerhan et al. 2015). Yet, as illustrated in Fig. 5, the $B-V$ colour of $0.38 \pm 0.06$ mag is consistent with no change from the precursor value of $0.39 \pm 0.02 \mathrm{mag}$, while the $V-I$ colour of $0.95 \pm 0.05$ mag has become only slightly redder from the precursor value of $0.81 \pm 0.01 \mathrm{mag}$.

\footnotetext{
${ }^{2}$ http://irsa.ipac.caltech.edu/data/SPITZER/docs/irac/
}

The latest epoch of near-IR $H$ and $K$ photometry from Kankare et al. (2015) nearly coincides with our HST UV-optical data from days 369-374 and Spitzer mid-IR photometry from 325. We thus combined these data to construct a spectral energy distribution (SED) for the source, shown in Fig. 6; the strong IR component of the SED is clearly seen.

\subsection{Dust modelling}

The Spitzer data were analysed under the assumption that the source of the mid-IR emission is hot dust. We fit the SED using simple models for graphite and silicate composition (Fox et al. 2010, 2011), with dust mass $\left(M_{\mathrm{d}}\right)$ and temperature $\left(T_{\mathrm{d}}\right)$ as free parameters. The flux is given by

$F_{v}=\frac{M_{\mathrm{d}} B_{v}\left(T_{\mathrm{d}}\right) \kappa_{v}(a)}{d^{2}}$

where $\kappa_{v}(a)$ is the dust absorption coefficient as a function of grain radius, and $d$ is the distance of the dust from the observer (Hildebrand 1983). We performed our calculations for grain sizes in the range $0.1-1.0 \mu \mathrm{m}$, looking up their associated $\kappa_{v}(a)$ values from the Mie scattering derivations discussed by Fox et al. (2010, see their fig. 4). For simplicity, we assume optically thin dust of a constant grain radius and emitting at a single equilibrium temperature (e.g. Hildebrand 1983). The data were fit using the IDL routine MPFIT. Table 3 lists the best-fitting parameters for $T_{\mathrm{d}}, M_{\mathrm{d}}$ and the dust luminosity $L_{\mathrm{d}}$ for graphite and silicates over a range of grain radii, for epochs $104 \mathrm{~d}$ through $328 \mathrm{~d}$. The average statistical uncertainties for $M_{\mathrm{d}}, T_{\mathrm{d}}$ and $L_{\mathrm{d}}$ are estimated at $\sim 30$ per cent, $\sim 25$ per cent and $\sim 30$ per cent, respectively. This estimate was obtained by performing several fits on the Spitzer data after offsetting the photometry by the photometric errors.

For our earliest epoch just before the onset of the main eruption, $30 \mathrm{~d}$ before peak, satisfactory model fits for $M_{\mathrm{d}}$ and $T_{\mathrm{d}}$ were not obtainable, limiting the luminosity to $L<2 \times 10^{6} \mathrm{~L} \odot$. For the successfully modelled epochs thereafter, we measure no significant change with time for the dust parameters of a given model, within our quoted uncertainty ranges. For the models of graphite dust grains with $a=0.1$ and $0.3 \mu \mathrm{m}$, the temperature remains 


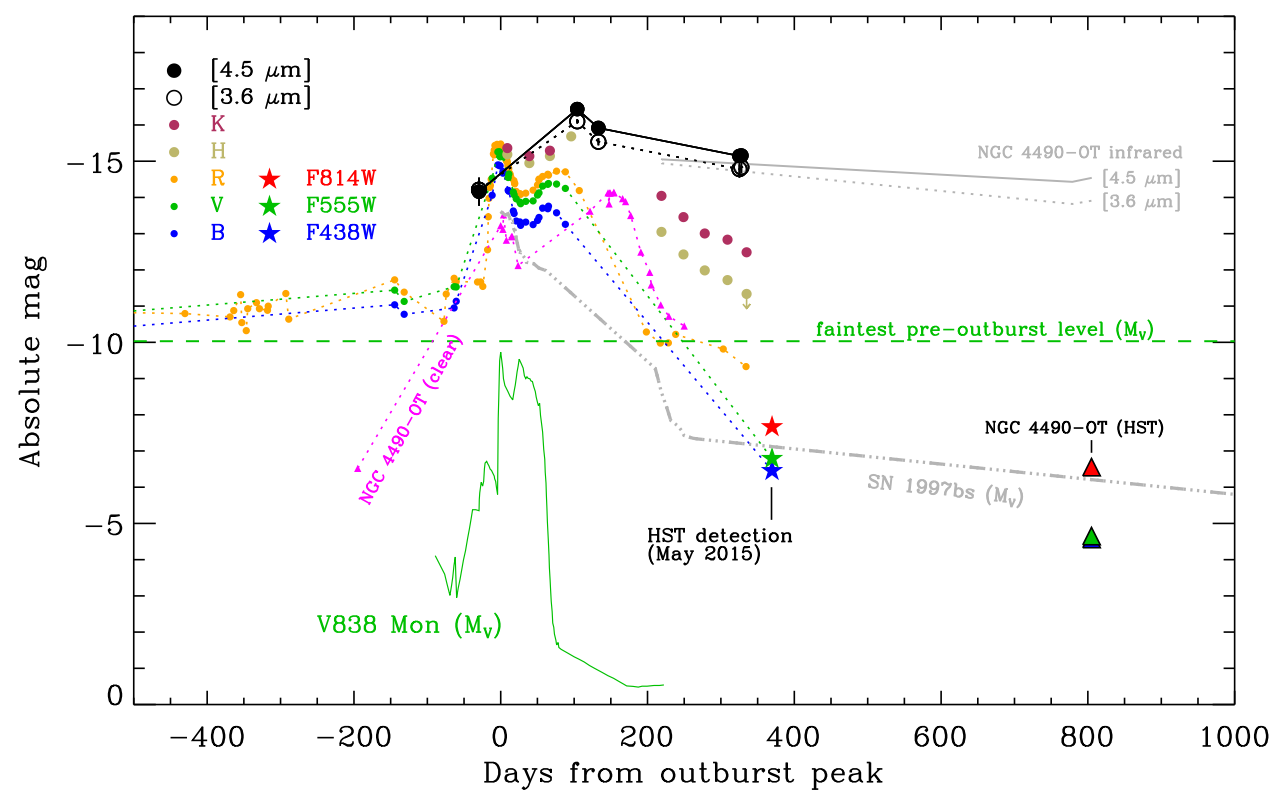

Figure 4. Long-term light curve of SN Hunt 248 (coloured filled circles), including the late-time mid-IR Spitzer (black open and filled circles) and UV-optical HST data (coloured five-pointed stars) presented here. Optical and near-IR photometric data of the precursor and main outburst are from Mauerhan et al. (2015) and Kankare et al. (2015). The optical photometry of NGC 4490-OT is also shown as coloured triangles (magenta is ground-based clear-filter photometry; red, green and blue are (respectively) late-time $F 814 \mathrm{~W}, F 555 \mathrm{~W}$ and $F 438 \mathrm{~W}$ filter photometry from HST; see Smith et al. 2016b); mid-IR Spitzer data on NGC 4490-OT are shown as grey solid and dotted curves. The optical light curve of SN 1997bs is displayed for comparison, including SN 1997bs (dashed triple-dotted grey curve; Van Dyk et al. 2000). The green horizontal dashed line represents the faintest pre-outburst $V$-band absolute magnitude of the precursor star (see Mauerhan et al. 2015). The V-band light curve of the purported stellar merger V838 Mon is also shown (green solid curve; Bond et al. 2003).

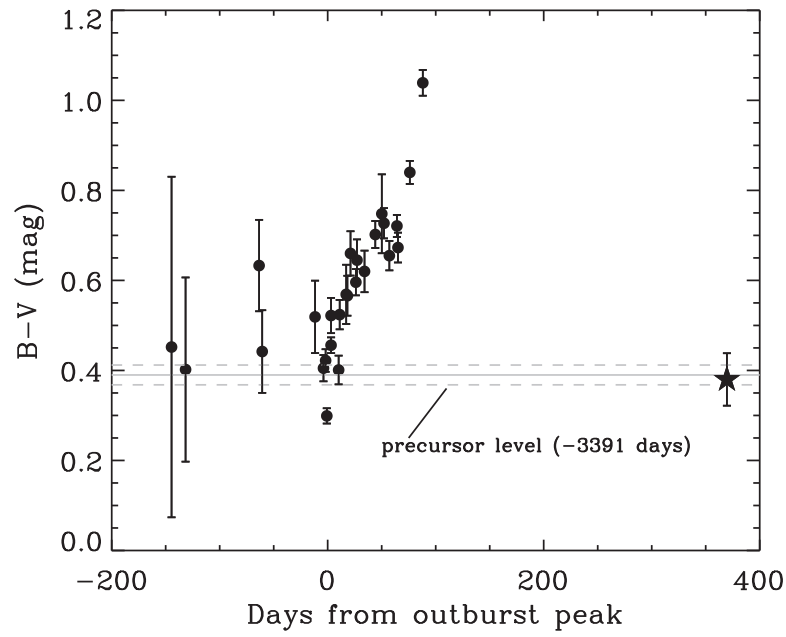

Figure 5. $B-V$ colour evolution of SN Hunt 248. The horizontal lines represent the value (thick line) and uncertainty envelope (thinner lines) of the stellar precursor detected with $H S T$ at $-3391 \mathrm{~d}$ (see Mauerhan et al. 2015). Filled dots are data from Kankare et al. (2015). The filled five-pointed star symbol represents our most recent measurement from Table 1, which exhibits the same value as the stellar precursor.

$800-900 \mathrm{~K}$, and inferred dust masses range between $\sim 3 \times 10^{-6} \mathrm{M}_{\odot}$ and $\sim 3 \times 10^{-5} \mathrm{M}_{\odot}$, with luminosities of the order of a few $\times 10^{6} \mathrm{M}_{\odot}$. For larger grain sizes of $a=0.5 \mu \mathrm{m}$ up to $1 \mu \mathrm{m}$, the range of potential temperatures is hotter $(1024-1720 \mathrm{~K})$. The masses of these larger grain models are systematically lower by a factor of a few, while the luminosities are comparable to those of the smaller grain models. For silicate grains, the model masses, temperatures and luminosities are all slightly higher than for graphite most notably for dust mass. However, the temperatures of the larger

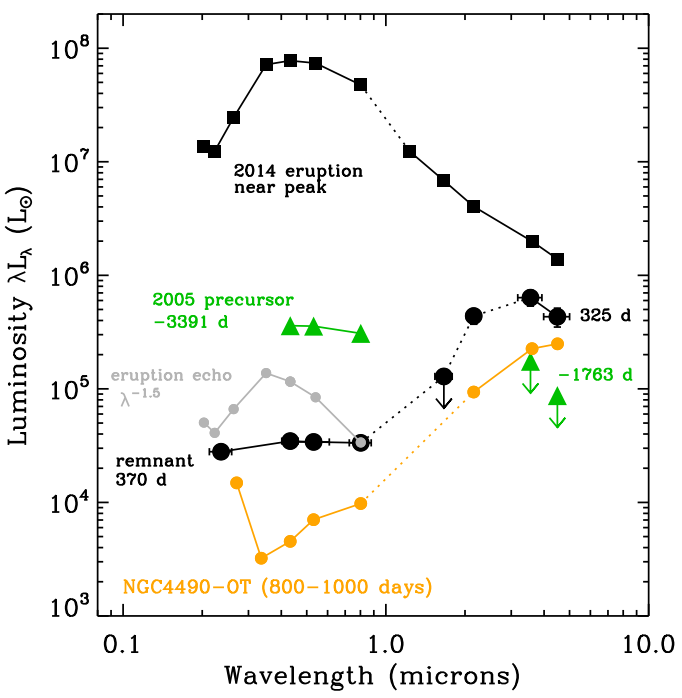

Figure 6. UV-IR SED of SN Hunt 248, including the 2014 outburst (black squares), the precursor (green triangles) and the remnant (black circles). The expected UV-optical SED of an echo of the 2014 eruption (see the text) is shown as grey circles. The SED of massive stellar merger candidate NGC 4490-OT is also shown for comparison (orange filled circles; data from Smith et al. 2016a, and reddened by adopting their extinction estimate with the extinction relation of Cardelli, Clayton \& Mathis 1989).

grain silicates are comparable to those of the smaller grain graphite models. We note, however, that $M_{\mathrm{d}}$ should probably be regarded as a lower limit, since there might also be a cooler component of dust to which our Spitzer observations at $3.6 \mu \mathrm{m}$ and $4.5 \mu \mathrm{m}$ are not sensitive. 
Table 3. Dust-model parameters for SN Hunt 248 at epochs $104-328$ d post-peak $^{a}$.

\begin{tabular}{|c|c|c|c|c|c|c|}
\hline$a(\mu \mathrm{m})$ & $T_{\mathrm{d}}(\mathrm{K})$ & $\begin{array}{r}\text { Graphite } \\
M_{\mathrm{d}}\left(\mathrm{M}_{\odot}\right)\end{array}$ & $L_{\mathrm{d}}\left(\mathrm{L}_{\odot}\right)$ & $T_{\mathrm{d}}(\mathrm{K})$ & $\begin{array}{r}\text { Silicates } \\
M_{\mathrm{d}}\left(\mathrm{M}_{\odot}\right)\end{array}$ & $L_{\mathrm{d}}\left(\mathrm{L}_{\odot}\right)$ \\
\hline \multicolumn{7}{|c|}{$104 \mathrm{~d}$} \\
\hline 0.10 & 868 & $2.7 e-5$ & $3.0 \mathrm{e}+6$ & 1247 & $4.1 \mathrm{e}-5$ & $5.5 \mathrm{e}+6$ \\
\hline 0.30 & 865 & $9.4 \mathrm{e}-6$ & $2.6 \mathrm{e}+6$ & 1140 & $4.9 \mathrm{e}-5$ & $5.5 \mathrm{e}+6$ \\
\hline 0.50 & 1086 & $3.6 e-6$ & $2.6 \mathrm{e}+6$ & 1038 & $5.6 e-5$ & $5.2 \mathrm{e}+6$ \\
\hline 0.75 & 1636 & $1.6 e-6$ & $4.7 e+6$ & 940 & $6.4 \mathrm{e}-5$ & $4.5 \mathrm{e}+6$ \\
\hline 1.00 & 1670 & $5.9 \mathrm{e}-7$ & $5.2 \mathrm{e}+6$ & 856 & $7.3 e-5$ & $3.7 \mathrm{e}+6$ \\
\hline \multicolumn{7}{|c|}{$133 \mathrm{~d}$} \\
\hline 0.10 & 830 & $2.0 \mathrm{e}-5$ & $1.7 \mathrm{e}+6$ & 1071 & $3.1 \mathrm{e}-5$ & $3.2 \mathrm{e}+6$ \\
\hline 0.30 & 827 & 7.0e-6 & $1.5 \mathrm{e}+6$ & 981 & $3.7 e-5$ & $3.2 \mathrm{e}+6$ \\
\hline 0.50 & 1024 & $2.7 e-6$ & $1.5 \mathrm{e}+6$ & 894 & $4.2 \mathrm{e}-5$ & $3.0 \mathrm{e}+6$ \\
\hline 0.75 & 1487 & $1.2 \mathrm{e}-6$ & $2.4 \mathrm{e}+6$ & 819 & $4.8 \mathrm{e}-5$ & $2.7 \mathrm{e}+6$ \\
\hline 1.00 & 1514 & $1.6 e-6$ & $2.6 \mathrm{e}+6$ & 870 & $5.4 \mathrm{e}-5$ & $2.3 e+6$ \\
\hline \multicolumn{7}{|c|}{$325 \mathrm{~d}$} \\
\hline 0.10 & 846 & $9.0 \mathrm{e}-6$ & $8.8 \mathrm{e}+5$ & 1199 & $1.4 \mathrm{e}-5$ & $1.6 \mathrm{e}+6$ \\
\hline 0.30 & 843 & $3.2 \mathrm{e}-6$ & $7.5 e+5$ & 1101 & $1.7 \mathrm{e}-5$ & $1.6 e+6$ \\
\hline 0.50 & 1050 & $1.2 \mathrm{e}-6$ & $7.6 e+5$ & 1006 & $1.9 \mathrm{e}-5$ & $1.5 \mathrm{e}+6$ \\
\hline 0.75 & 1549 & $5.4 \mathrm{e}-7$ & $1.3 e+6$ & 914 & $2.2 e-5$ & $1.3 e+6$ \\
\hline 1.00 & 1579 & $7.2 \mathrm{e}-7$ & $1.4 \mathrm{e}+6$ & 835 & $2.5 e-5$ & $1.1 \mathrm{e}+6$ \\
\hline \multicolumn{7}{|c|}{$328 \mathrm{~d}$} \\
\hline 0.10 & 882 & $7.7 e-6$ & $9.7 \mathrm{e}+5$ & 1279 & $1.2 \mathrm{e}-5$ & $1.7 \mathrm{e}+6$ \\
\hline 0.30 & 879 & $2.7 e-6$ & $8.2 \mathrm{e}+5$ & 1166 & $1.4 \mathrm{e}-5$ & $1.7 \mathrm{e}+6$ \\
\hline 0.50 & 1109 & $1.0 \mathrm{e}-6$ & $8.3 e+5$ & 1059 & $1.6 e-5$ & $1.6 e+6$ \\
\hline 0.75 & 1697 & $4.4 \mathrm{e}-7$ & $1.6 \mathrm{e}+6$ & 957 & $1.9 e-5$ & $1.4 \mathrm{e}+6$ \\
\hline 1.00 & 1733 & $5.9 \mathrm{e}-7$ & $1.7 \mathrm{e}+6$ & 870 & $2.1 \mathrm{e}-5$ & $1.2 \mathrm{e}+6$ \\
\hline
\end{tabular}

Note. ${ }^{a}$ Only upper limits on dust parameters were obtainable for our earliest epoch at $-30 \mathrm{~d}$ post-peak (not listed; see the text). Average uncertainties for fit parameters $M_{\mathrm{d}}, T_{\mathrm{d}}$ and $L_{\mathrm{d}}$ are estimated at 30 per cent, 25 per cent and 30 per cent, respectively (see the text).
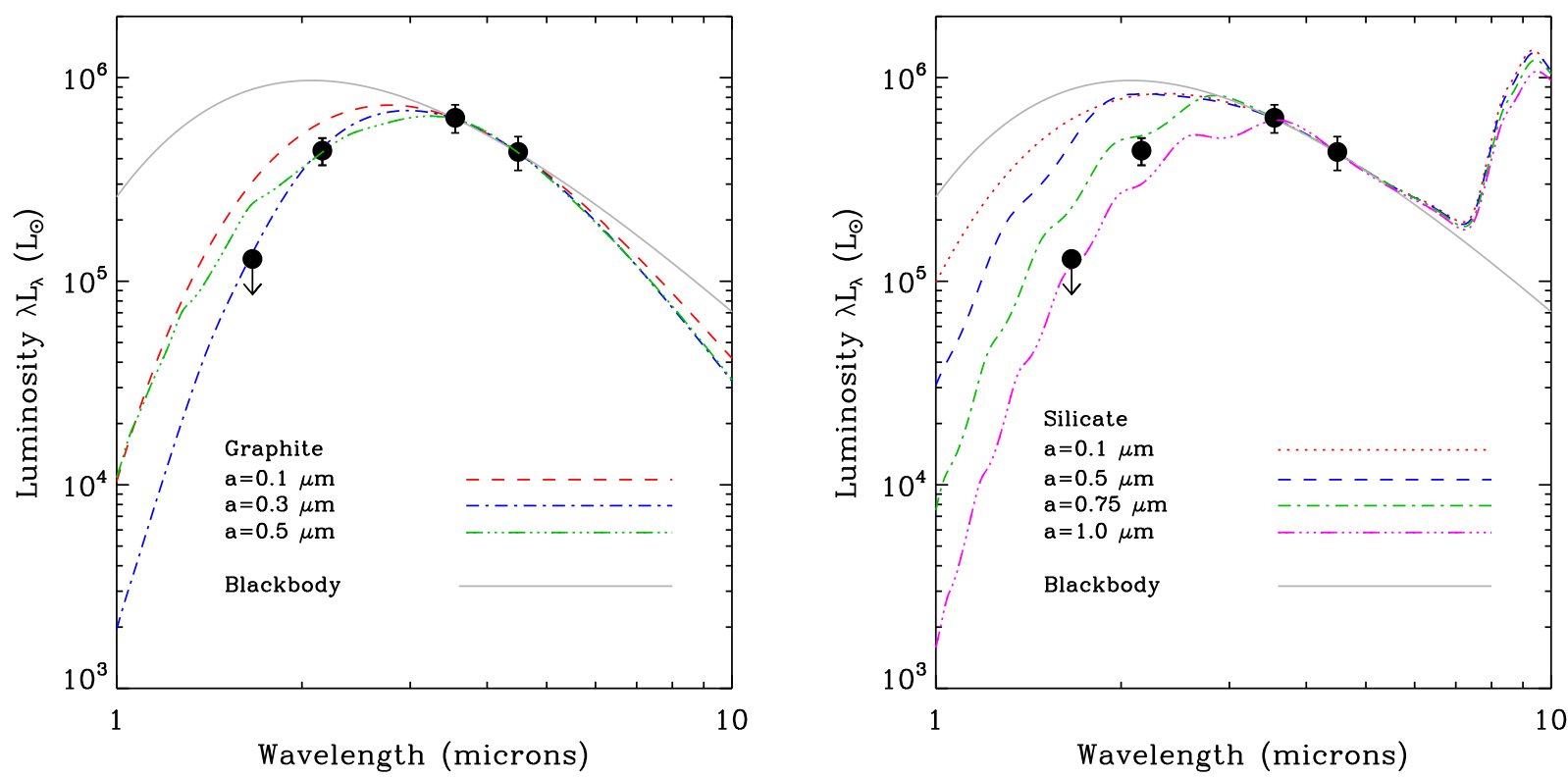

Figure 7. Infrared photometry of SN Hunt 248 on day 328 post-peak compared with the SEDs of graphite (left-hand panel) and silicate (right-hand panel) model dust sources. $H$ and $K$ points are day-332 measurements from Kankare et al. (2015).

Although our model parameters were fit using only the Spitzer photometry at 3.6 and $4.5 \mu \mathrm{m}$, the epoch on day 328 post-peak was only $5 \mathrm{~d}$ before a ground-based near-IR $H$ and $K$ measurement from Kankare et al. (2015), so we used those data to further discriminate between the various SED models. This last epoch is also particularly important in that it is close in time to our UV-optical HST photometry of the remnant, and so can be used to estimate the expected UV-optical extinction from the dust parameters we derived (see Section 4.1.2). As shown in Fig. 7, the results suggest that the average grain size for both the silicate and graphite models 
is likely to be substantially larger than the $0.1 \mu \mathrm{m}$ average grain size. Indeed, simple blackbody distributions of any temperature are too broad to fit the SED of SN Hunt 248, and dust models for small grain sizes are also too broad and significantly overestimate the flux in the near-IR; the source is clearly a grey body. For graphite, the SED appears most consistent with $0.3 \mu \mathrm{m}$ grains, while for silicate dust, even larger grain sizes in the range $0.75-1.0 \mu \mathrm{m}$ appear to provide the best match to the day 328 data.

The size of the emitting region can be estimated by considering the radius of an equivalent blackbody having luminosity and temperature indicated by the model fits,

$r_{\mathrm{bb}}=\left(\frac{L_{\mathrm{d}}}{4 \pi \sigma T_{\mathrm{d}}^{4}}\right)^{1 / 2}$.

Focusing on the last epoch at $328 \mathrm{~d}$, the best-matching graphite $(a=0.3 \mu \mathrm{m})$ and silicate $(a=0.75-1.0 \mu \mathrm{m})$ dust models indicate respective radii of $2.7 \times 10^{15} \mathrm{~cm}$ and $(3.1-3.3) \times 10^{15} \mathrm{~cm}$. We therefore assume an approximate value of $3 \times 10^{15} \mathrm{~cm}$ for the following analysis and interpretation.

\section{DISCUSSION}

\subsection{The nature of the remnant}

\subsubsection{The origin of the dust}

The SED of the UV-IR remnant of SN Hunt 248, shown in Fig. 6, appears very similar to that of NGC 4490-OT (Smith et al. 2016b). In both cases, the dust is hot and emits like a grey body, and the UV-optical counterpart has noteworthy UV flux, especially NGC 4490-OT. As shown in Figs 4 and 6, the mid-IR brightness evolution of both objects exhibits very similar plateaus, and they both have IR luminosities that are comparable to the optical luminosities of their directly identified stellar precursors; taken at face value, this appears consistent with heating of the dust by a luminous surviving star. Interestingly, the estimated radius of the dust $\left(3 \times 10^{15} \mathrm{~cm}\right)$ matches the expected expansion radius of the ejecta after $328 \mathrm{~d}$, considering the measured outflow speed of $v \approx 1200 \mathrm{~km} \mathrm{~s}^{-1}$ (Mauerhan et al. 2015). Thus, the measurements seem consistent with dust condensation in the ejecta from the 2014 event.

Alternatively, pre-existing dust may have been swept to large radius by the ejecta. After all, the spectra near peak brightness did exhibit the signatures of CSM interaction (see Mauerhan et al. 2015). Assuming a gas-to-dust ratio of 100, the dust masses inferred by our model $\left(\sim 10^{-6}\right.$ to $\left.10^{-5} \mathrm{M}_{\odot}\right)$ imply a total CSM mass of $\sim 10^{-4}$ to $10^{-3} \mathrm{M}_{\odot}$. Therefore, if the 2014 eruption ejected only $0.1 \mathrm{M}_{\odot}$ (which would be modest compared to the $>10 \mathrm{M}_{\odot}$ ejected by $\eta$ Car's historic event), the pre-existing CSM would not be massive enough to effectively decelerate the ejecta. Dust in the circumstellar environment could therefore have been swept to the expansion radius, if the grains survived the UV radiation and shock of the event. We speculate that this might explain the relatively large sizes of dust grains inferred by our models for the IR emission - i.e. the smallest circumstellar grains could have been destroyed by the 2014 outburst, leaving a distribution skewed towards larger sizes.

Unfortunately, our Spitzer mid-IR upper limits on the stellar precursor are not sufficiently deep to provide a meaningful constraint on the pre-existing dust mass, so we cannot tell if the dust mass was lower before the eruption than in the aftermath. For example, Fig. 8 shows the SED of the precursor to SN Hunt 248 along with those of several Galactic cool hypergiants which have measured

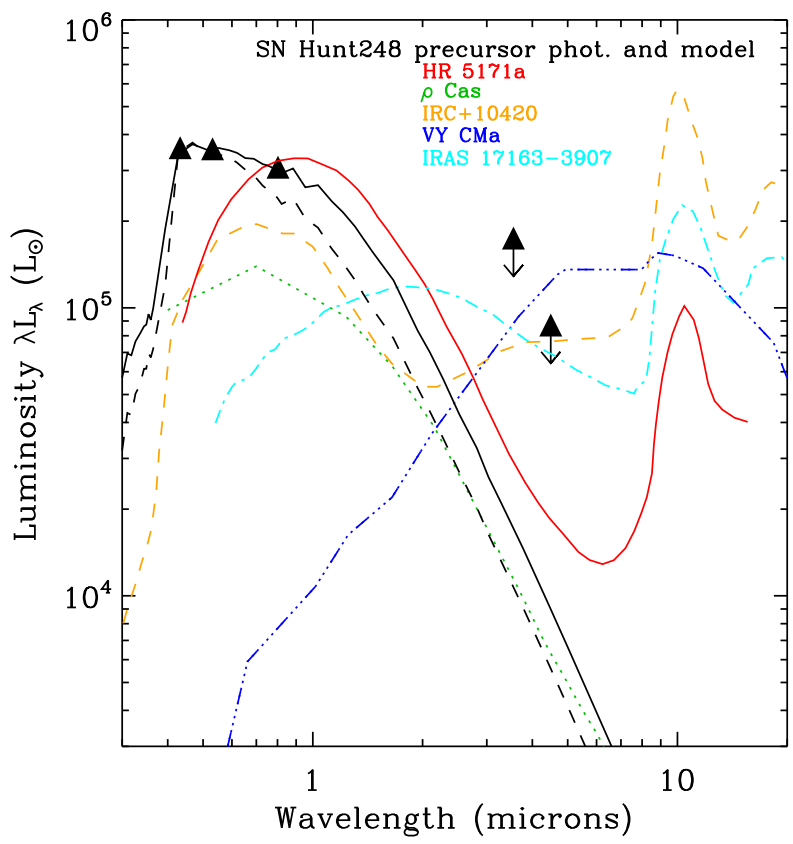

Figure 8. Optical luminosity of the stellar precursor of SN Hunt 248 (filled triangles; Mauerhan et al. 2015) and its mid-IR limits (black filled triangles with downward-facing arrows). The photometry was corrected only for interstellar extinction $\left(A_{V}=0.14 \mathrm{mag}, R_{V}=3.1\right)$. The solid black curve is the SED of a star with $T_{\text {eff }}=7000 \mathrm{~K}$ (Castelli \& Kurucz 2003), scaled to $L / \mathrm{L}_{\odot}=6.07$, and reddened by an additional component of grey circumstellar extinction $\left(A_{V}=0.86 \mathrm{mag}, R_{V}=5.4\right)$. The SED equivalent to the previously estimated stellar parameters from Mauerhan et al. (2015), which did not account for circumstellar extinction, is represented by the black dashed curve. For comparison purposes, we also show the SEDs for the Galactic cool-warm hypergiants VY CMa, $\rho$ Cas and IRC+10420 (blue dashed triple-dotted, green dotted and orange dashed curves, respectively; Shenoy et al. 2016), HR5171a (red solid curve; Humphreys, Strecker \& Ney 1971) and IRAS 17163-3907 (cyan dash-dotted curve; Lagadec et al. 2011). The following distances were used to calculate the luminosity: SN Hunt 248 (26.4 Mpc; Mauerhan et al. 2015), $\rho$ Cas (2.5 kpc; Humphreys 1978); VY CMa (1.2 kpc; Shenoy et al. 2016), IRC+10420 ( $5 \mathrm{kpc}$; Shenoy et al. 2016), HR5171 (3.6 kpc; Chesneau et al. 2014a) and IRAS $17163-3907$ (4.2 kpc, average of range estimate from Lagadec et al. 2011). The IRAS 17163-3907 data were corrected for interstellar extinction in this work, adopting $A_{V}=2.1 \mathrm{mag}$ (Lagadec et al. 2011) and the extinction relation of Cardelli, Clayton \& Mathis (1989). The other SEDs from the literature account only for interstellar extinction.

circumstellar dust masses in the literature. Our limits are mutually consistent with a system like $\rho$ Cas, which has a rather low estimated dust mass of $\sim 3 \times 10^{-8} \mathrm{M}_{\odot}$ (Jura \& Kleinmann 1990), and more extreme dusty systems like IRC +10420 (Shenoy et al. 2016) and IRAS 17163-3907 (Lagadec et al. 2011), the latter of which has a much larger dust mass of $\sim 0.04 \mathrm{M}_{\odot}$. The comparison in Fig. 8 does, however, suggest that the IR excess from a system such as VY CMa, with a total dust mass of $\sim 0.02 \mathrm{M}_{\odot}$ (Harwit et al. 2001; Muller et al. 2007), would have been detectable at $4.5 \mu \mathrm{m}$. It is therefore plausible that the $\sim 10^{-5} \mathrm{M}_{\odot}$ dust mass we inferred post eruption could have been pre-existing, yet not detectable by our Spitzer observations.

Finally, we should address the possibility that the IR (and perhaps optical) emission of the remnant is the result of a light echo of the 2014 outburst off of outer dusty CSM. In such a scenario, there is both delayed scattering of UV-optical light and thermal IR reprocessing of the fraction of light which gets absorbed by 
the dust. However, assuming that such an echo is dominated by light from the peak of the outburst and obeys a $\propto \lambda^{-1.5}$ wavelength dependence (e.g. see Fox et al. 2015), while suffering the same extinction as the precursor, the expected UV-optical SED is totally inconsistent with the observed SED at $+370 \mathrm{~d}$ (see Fig. 6, grey curve). The thermal-IR remnant also appears to be inconsistent with thermal reprocessing of an echo, as the dust temperature requires a luminosity that is far above the peak of the 2014 event. This was determined using the same line of reasoning invoked for the analysis of the remnant of NGC 4490-OT (see Smith et al. 2016a, their section 3.2.3). Assuming the ratio of the efficiencies of UV absorption to IR emission as $Q_{\mathrm{UV}} / Q_{\mathrm{IR}}=0.3$ (Smith et al. 2016b), the luminosity required to heat dust at a distance $r$ to a temperature $T$ can be expressed as $L / \mathrm{L}_{\odot} \approx 5.7 \times 10^{12}\left(T_{\mathrm{d}} / 1000 \mathrm{~K}\right)^{4}(r / \mathrm{pc})^{2}$. At $328 \mathrm{~d}$, the minimum distance of the echo-heated dust is $r \approx 0.3 \mathrm{pc}$. Thus, the range of possible dust temperatures inferred from our model fits and their uncertainties $(650-1450 \mathrm{~K})$ requires a peak outburst luminosity of $(1-25) \times 10^{11} \mathrm{~L}_{\odot}$, which is three orders of magnitude higher than the observed peak of the 2014 outburst. Furthermore, the temperature evolution of a thermally reprocessed echo is expected to evolve with time as $T \propto t^{-0.5}$ (Fox et al. 2011, 2015), and so we would have expected the temperature between 133 and $325 \mathrm{~d}$ to have dropped from $\sim 830$ to $\sim 60 \mathrm{~K}$; instead, the temperature evolution is consistent with no change between these epochs. We therefore conclude that a light echo is inconsistent with the available data, and therefore is not the source of the late-time UV-optical source and its thermal counterpart. The hypotheses of dust synthesis in the ejecta and swept-up CSM dust are far more consistent with the data.

\subsubsection{Circumstellar extinction and intrinsic stellar parameters}

If the UV-optical component of the SED is from a surviving star and the thermal emission is from circumstellar dust that absorbs stellar radiation, then we should consider the potential effect of dust absorption on the optical properties of the remnant. Under the assumption of a spherically symmetric shell geometry of thickness $\Delta r$, the optical depth of the dust at a given wavelength can be expressed by

$\tau_{\lambda}=\kappa_{\lambda}(a) \rho \Delta r=\kappa_{\lambda}(a) \frac{M_{\mathrm{d}}}{4 \pi r_{\mathrm{bb}}^{2}}$,

where $\rho$ is the density of the dust shell and $\kappa_{\lambda}(a)$ is the absorption coefficient for the dust of a particular grain radius and at a particular wavelength. The $V$-band $(\lambda=0.555 \mu \mathrm{m})$ absorption coefficients for our best-matching graphite $(a=0.3 \mu \mathrm{m})$ and silicate $(a=0.75-1.0 \mu \mathrm{m})$ models are $\sim 14700$ and $\sim 2000-2600 \mathrm{~cm}^{-2} \mathrm{~g}^{-1}$, respectively (see Fox et al. 2010, their fig. 4). Using the model dust masses in Table 3 and the radius of $3 \times 10^{15} \mathrm{~cm}$ derived in Section 3.1, we estimate $V$-band optical depths of $\tau_{V} \approx 0.9$ for graphite and $\sim 0.7-0.9$ for silicates. If we ignore the effect of grain albedo and optical scattering for the moment, then the extinction can be approximated by $1.086 \tau$, in which case we obtain $A_{V} \approx 0.8-1$ mag. The total extinction from the ISM and hot-dust component would therefore be approximately the same for both the graphite and silicate models, with $A_{V} \approx 1.0 \mathrm{mag}$. This implies $M_{V} \approx-7.6 \mathrm{mag}$ for the remnant and, thus, supergiant luminosity class. However, a more realistic treatment of the effective extinction accounts for the scattering albedo, $\omega$, of the grains: $A=1.086(1-\omega)^{1 / 2} \tau_{V}$. For a standard ISM-like distribution of graphitic (silicate) grains, the scattering albedo is $0.5(0.9)$ and would thus reduce the required extinction to 0.8 (0.4) mag (Kochanek, Khan \& Dai 2012a). However, for large grains with $a=0.3-1.0 \mu \mathrm{m}$, the effective albedo

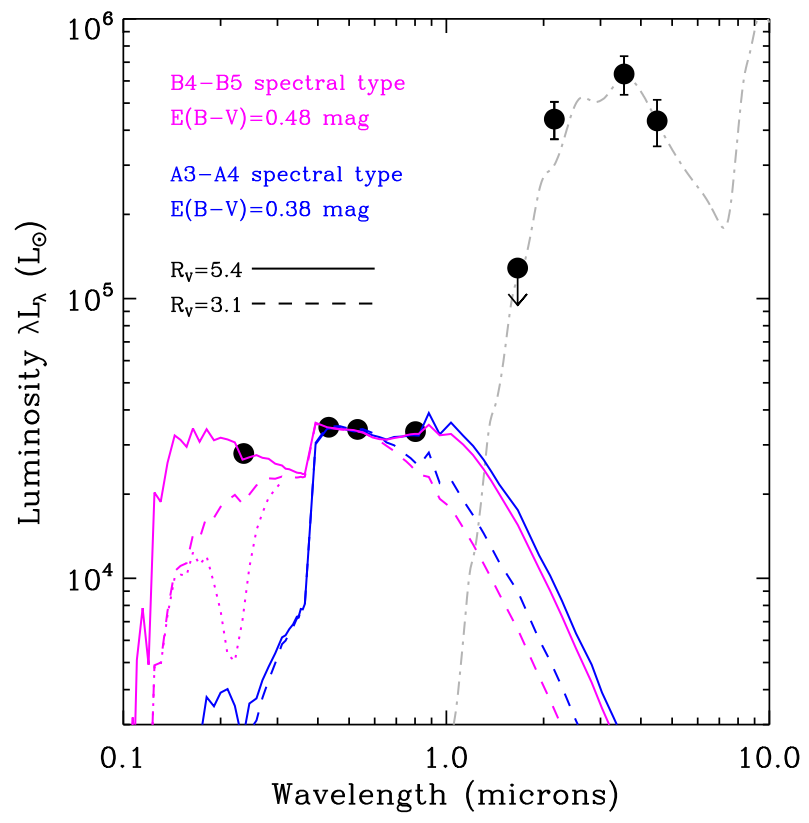

Figure 9. SED of the SNHunt 248 optical (infrared) remnant at 370 (325) d (black filled circles; corrected only for interstellar extinction $A_{V}=0.14 \mathrm{mag}$ and $R_{V}=3.1$ ). The magenta curves are the SED of a model $T_{\text {eff }}=15000 \mathrm{~K}$ star (spectral type B4-B5; Castelli \& Kurucz 2003), reddened by $E(B-V)=0.48$ mag (to illustrate the effect of circumstellar extinction) for two different extinction laws. The solid (dashed) curves represent extinction laws having $R_{V}=5.4(3.1)$. The reddened models have been vertically scaled to match the $B$ and $V$ photometry. The blue curves are for a model SED of a $T_{\text {eff }}=8500 \mathrm{~K}$ star (spectral type A3-A4) reddened by $E(B-V)=0.38 \mathrm{mag}$, shown to demonstrate that cooler models greatly underestimate the UV photometry. For reference, the dotted magenta curve near $0.2 \mu \mathrm{m}$ wavelength shows the effect which a Galactic interstellar UV opacity bump would have on the B4-B5, $E(B-V)=0.48 \mathrm{mag}, R_{V}=3.1$ model. Our silicate IR emission model for $a=1.0 \mu \mathrm{m}$ is also shown (dashdotted grey curve).

could be considerably lower $(\omega \approx 0.1$; Mulders et al. 2013) and thus scattering might have only a small impact on the effective extinction. Without reliable information on the albedo of the grains, all we can say is that the expected extinction from the same hot-dust component which is responsible for the IR emission is $A_{V}<1$ mag.

The effect of the estimated extinction on the colours of the star depends on the assumed value of total-to-selective extinction $R_{V}$, defined as $A_{V} / E(B-V)$, which is sensitive to the dust chemistry and grain-size distribution. If we were to hypothetically assume $A_{V}=1 \mathrm{mag}$ and an ISM-like value of $R_{V}=3.1$ for SN Hunt 248, then the associated $E(B-V) \approx 0.3 \mathrm{mag}$ would imply an intrinsic colour in the range $(B-V)_{0}=0.1 \mathrm{mag}$, corresponding to a spectral type in the range A3-A4 (Fitzgerald 1970). However, such a spectral type provides a poor match to the UV-optical SED, as illustrated by Fig. 9. A3-A4 stars exhibit a relative UV luminosity that is an order of magnitude lower than that of the optical bands. On the contrary, the strong UV flux of the data indicates that the star is significantly hotter, with a substantial Balmer continuum flux. Specifically, after matching stellar model SEDs having a wide range of temperatures (from Castelli \& Kurucz 2003) and over a wide range of $A_{V}$ and $R_{V}$, we found that the best match to the four bands of our measured UVoptical SED is provided by a star with $T_{\text {eff }}=15000 \mathrm{~K}$ (appropriate for a B4-B5 star of supergiant luminosity class; Zorec et al. 2009) with extinction parameters $A_{V}=2.6 \mathrm{mag}$ and $R_{V}=5.4$ (with no ISM-like UV 'bump' in the extinction law). Cooler models cannot 
supply enough Balmer continuum, while hotter stellar SEDs with $T_{\text {eff }}>15000 \mathrm{~K}$ produce too much UV flux, and cannot provide a good match for any of the wide range of $A_{V}$ and $R_{V}$ values we attempted. We conservatively estimate a temperature uncertainty of $\delta T=1000 \mathrm{~K}$ for the remnant star.

Clearly, the extinction value of $A_{V}=2.6 \mathrm{mag}$ implied by our best-matching stellar SED is significantly higher than our estimates of the expected absorption from the hot-dust component, which suggested $A_{V}<1 \mathrm{mag}$. However, a higher value of extinction would not be surprising, given that the hot dust responsible for the IR emission probably comprises only a fraction of the total dust mass; indeed, it is plausible that there is cooler dust in the system which does not emit strongly at 3-5 $\mu \mathrm{m}$. Furthermore, the value of $A_{V}=2.6 \mathrm{mag}$ implied by the SED would also explain the factor of $\sim 10$ drop in apparent brightness of the remnant star relative to the precursor. Meanwhile, the high $R_{V}$ we inferred from the SED might actually be appropriate for circumstellar dust having a grain distribution skewed towards large sizes. For example, $\sim 40$ per cent of the extinction in the interacting SN 2010jl has been attributed to large graphitic dust grains with maximum sizes above $a=0.5 \mu \mathrm{m}$ and possibly as large as $a>1.3 \mu \mathrm{m}$, which result in an estimated $R_{V}=6.4$ (Gall et al. 2014). In another example, observations of the red hypergiant VYCMa necessitate a circumstellar total-toselective extinction value of $R_{V}=4.2$ (Massey et al. 2005), also potentially the result of a grain distribution skewed towards larger sizes. In addition, large grains in $\eta$ Car's Homunculus nebula have been invoked to explain the apparently grey extinction of the central source (Andriesse, Donn \& Viotti 1978; Robinson et al. 1987; Davidson et al. 1999; Smith et al. 2000; Smith \& Ferland 2007; Kashi \& Soker 2008).

The integrated extinction-corrected luminosity of the bestmatching B4-B5 SED is $L \approx 1.2 \times 10^{6} \mathrm{~L} \odot$. We note that this is approximately twice the luminosity of our previous estimate for the cool hypergiant precursor (Mauerhan et al. 2015). However, that earlier work focused on matching stellar models to the $B$ and $V$ photometry alone (ignoring the poor fit to the $I$-band photometry) and assumed no circumstellar extinction. We thus revisited the precursor photometry in this work using stellar SED models from Castelli \& Kurucz (2003), reddened by an additional component of circumstellar extinction. We find that the best-matching stellar model is one in which there is substantial grey circumstellar extinction, similar to our conclusion for the hotter B4-B5 remnant. As shown in Fig. 8, we obtain a reasonable match to the $B, V$ and $I$ precursor photometry using a stellar model SED with $T_{\text {eff }}=7000 \mathrm{~K}$ (Castelli \& Kurucz 2003), reddened by $E(B-V)=0.16 \mathrm{mag}$ and $R_{V}=5.4$ ( $\left.A_{V}=0.86 \mathrm{mag}\right)$. This temperature is more or less equivalent to our previous estimate in Mauerhan et al. (2015), and thus remains consistent with the yellow (F-type) hypergiant classification. We note that if we had used a standard ISM-like $R_{V}=3.1$, then we achieve poor matches for a wide range of stellar models and extinction values. Moreover, stellar models with lower effective temperatures than $7000 \mathrm{~K}$ exhibit $B$-band fluxes well below the photometry. Based on our attempted matches to models with a variety of temperatures, we conservatively estimate a temperature uncertainty of $\delta T=1000 \mathrm{~K}$ for the precursor. The integrated unreddened luminosity of our best-matching stellar model (shown in Fig. 8) is also $L \approx 1.2 \times 10^{6} \mathrm{~L} \odot$, equivalent to that of the best-matching B4-B5 remnant model shown in Fig. 9. Taken at face value, this is consistent with a temperature change of $\delta T \approx 8000 \mathrm{~K}$ at constant luminosity of $\sim 1.2 \times 10^{6} \mathrm{~L} \odot$.

The revised luminosity estimate of the precursor warrants an examination of the star's associated transition in the HR diagram, which we show in Fig. 10. After correcting the precursor photometry

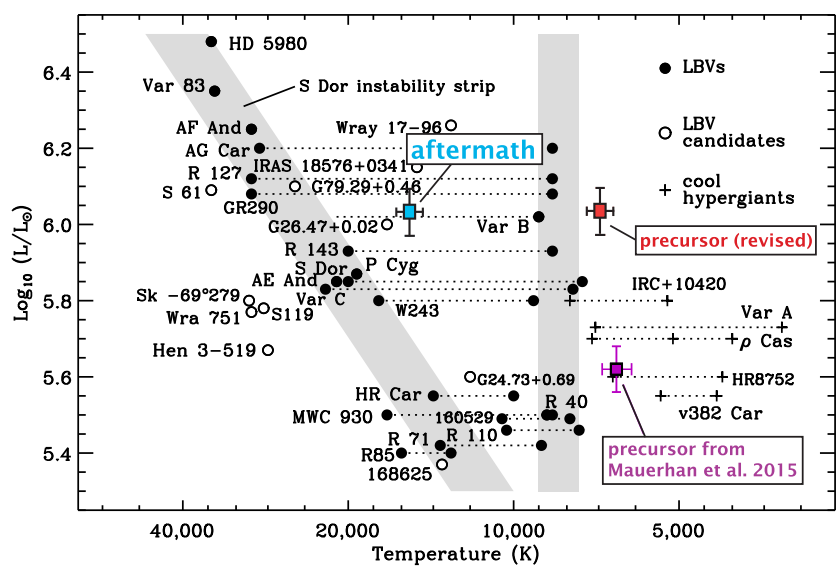

Figure 10. Modification of the HR diagram for LBVs and their kin from Mauerhan et al. (2015, see their fig. 8). The magenta coloured square is the previous estimate for the precursor star from Mauerhan et al. (2015), uncorrected for possible circumstellar extinction. The red square represents the revised best-matching model of the precursor SED, corrected for grey circumstellar extinction (see the text). The blue square indicates the aftermath of the eruption, a hot B4-B5 supergiant, also corrected for grey circumstellar extinction. The luminosities of the remnant and the revised precursor were calculated by integrating the UV-IR SEDs of the best-matching models shown in Figs 8 and 9.

for the circumstellar extinction discussed above $\left(A_{V}=0.86 \mathrm{mag}\right.$ and $R_{V}=5.4$ ), the precursor star would occupy a region more luminous than the cool hypergiants, yet still within the observed temperature range exhibited by stars of this class (note, however, that circumstellar extinction may not be adequately addressed in other objects classified as cool hypergiants). After the eruption, the hotter remnant has migrated bluewards, and lies in between the S Dor and red instability strips. Future observations will determine whether the remnant continues to migrate in the HR diagram towards the hotter S Dor instability strip occupied by quiescent LBVs, or if increasing extinction from ongoing dust condensation in the ejecta pushes it redwards again.

The revised parameters of the stellar precursor warrant re-analysis of the star's initial mass as well, which was previously estimated at $\sim 30 \mathrm{M}_{\odot}$ (Mauerhan et al. 2015). Fig. 11 shows the data, after correcting for the purported grey circumstellar extinction parameters discussed above (for the remnant, $E(B-V)=0.48$ mag and $R_{V}=5.4$; for the precursor, $E(B-V)=0.16 \mathrm{mag}$ and $R_{V}=5.4$ ), along with evolutionary tracks from the Geneva rotating stellar models for 50 and $60 \mathrm{M}_{\odot}$ (Ekström et al. 2012); the data appear to most closely match (but are slightly below) the $60 \mathrm{M}_{\odot}$ model, which at the locations of both the remnant and precursor is undergoing core-He burning (Ekström et al. 2012). This revised initial mass is approximately twice as high as the circumstellar extinction-free estimate by Mauerhan et al. (2015). Interestingly, the photometry of the remnant and precursor, which was corrected for different values of circumstellar extinction and based on the best SED matches, is consistent with no significant change in stellar luminosity between before and after the 2014 eruption.

\subsection{The 2014 outburst, revisited}

\subsubsection{Massive binary merger-burst?}

The energy source of the 2014 eruption is uncertain. The structure of the outburst light curve, the outflow velocity and the large-amplitude pre-outburst variability detected over prior decades might provide 


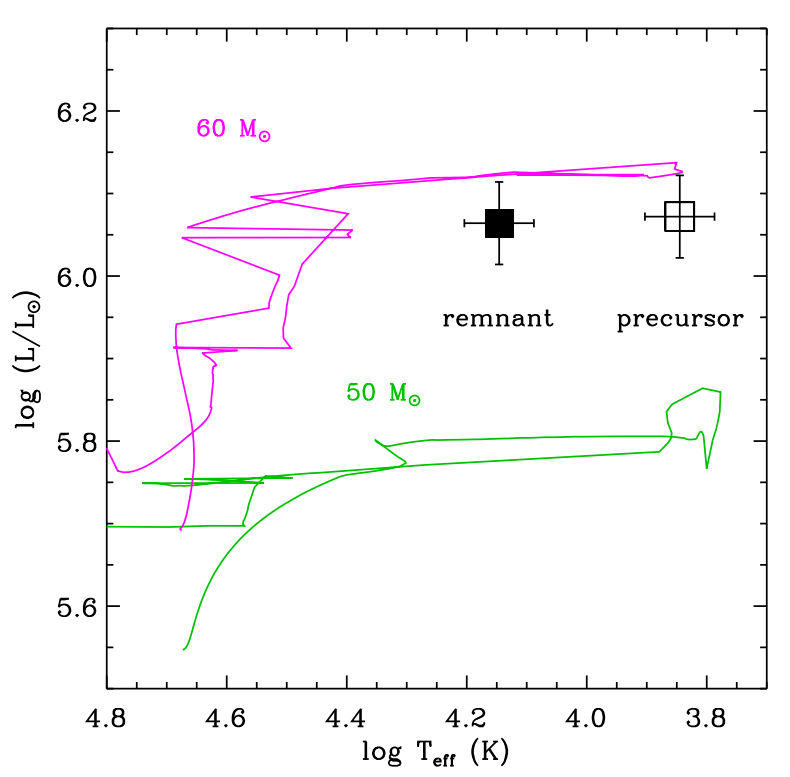

Figure 11. The precursor (open black square) and remnant (filled black square) photometry of SN Hunt 248 on the HR diagram, after correcting for our estimated circumstellar extinction parameters (see the text, Section 4.1.2). The evolutionary tracks are from Geneva rotating stellar models (Ekström et al. 2012) at solar metallicity for initial masses of $50 \mathrm{M}_{\odot}$ (green curve) and $60 \mathrm{M}_{\odot}$ (magenta curve).

clues. We can speculate that the cool-hypergiant precursor was a massive interacting binary, perhaps similar to HR 5171 (Chesneau et al. 2014a), and that its large pseudophotosphere was the signature of a common envelope. If this is the case, it is plausible that SN Hunt 248's 2014 eruption was driven by a violent binary encounter, common-envelope ejection or a merger-burst marking the coalescence of two massive stars (see Paczyński 1971; Vanbeveren, De Loore \& Van Rensbergen 1998, 2013; Podsiadlowski et al. 2010; Langer 2012; Justham, Podsiadlowski \& Vink 2014; Portegies-Zwart \& van den Heuvel 2016). Indeed, such events might be more common than previously thought (Kochanek, Adams \& Belczynski 2014), and their transients might actually explain a substantial fraction of SN impostors and LBV eruptions, including the historic outburst of $\eta$ Car (Portegies Zwart \& van den Heuval 2016).

Fig. 12 shows the peak outburst luminosity versus outflow velocity ${ }^{3}$ for SN Hunt 248 (Mauerhan et al. 2015); NGC 4490-OT (Smith et al. 2016b); the sample of merger candidates presented by Pejcha, Metzger \& Tomida (2016a, their fig. 21); M101-OT, also considered a merger candidate or binary common-envelope ejection event (Blagorodnova et al. 2017); and $\eta$ Car (Smith et al. 2003; Smith \& Frew 2011). Interestingly, SN Hunt 248 is consistent with the apparent trend exhibited by this sample of merger candidates. Smith et al. (2016b) interpreted NGC 4490-OT as a stellar merger involving a star of similarly high mass to SN Hunt $248\left(\sim 30 \mathrm{M}_{\odot}\right)$, and reiterated the suggestion that $\eta$ Car's historic eruption was the result of a massive merger. Indeed, $\eta$ Car's position in Fig. 12 also fits in with the apparent trend exhibited by other merger candidates. V1309 Sco was almost certainly a true merger, based on

\footnotetext{
${ }^{3}$ We note that the outflow velocities of SN Hunt 248 and NGC4490-OT were measured from their PCygni absorption minima (Mauerhan et al. 2015; Smith et al. 2016b), whereas the outflow velocities of the sample in Pejcha, Metzger \& Tomida (2016a) were measured mostly by $\mathrm{H} \alpha$ line widths, and $\eta$ Car's velocity measurement is from detailed spectroscopic analysis of the Homunculus nebula (Smith et al. 2003).
}

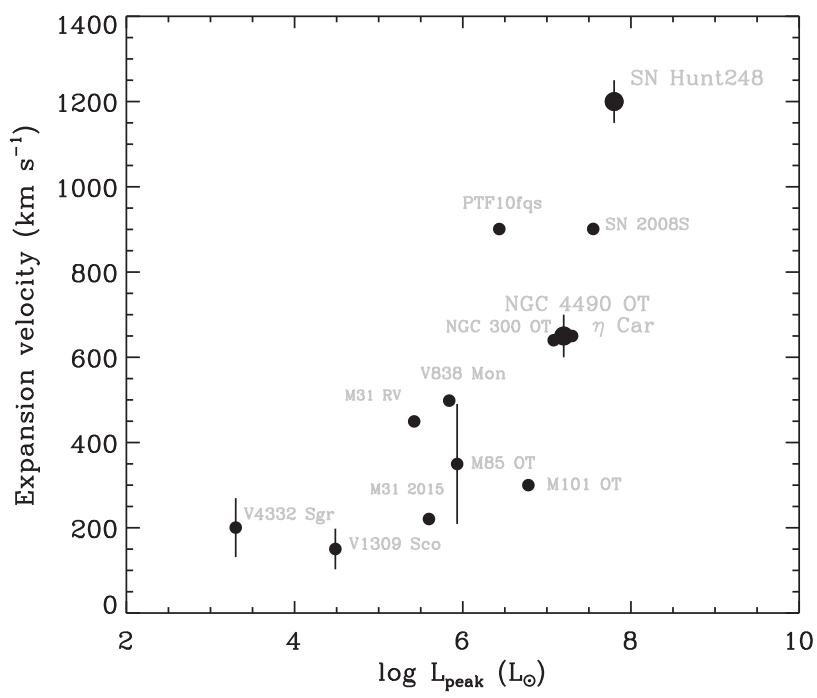

Figure 12. Peak outburst luminosity versus outflow velocity for SN Hunt 248 and other stellar merger candidates, including NGC 4490-OT (Smith \& Tombleson 2015) and the sample presented by Pejcha, Metzger $\&$ Tomida (2016a, their fig. 21); $\eta$ Car is also included. Uncertainties in expansion velocity are shown where available.

the exquisite light curve that showed the rapidly decreasing orbital period of an inspiralling binary (Tylenda et al. 2011). V838 Mon was thought to be a similar merger involving a B-type star (Tylenda, Soker \& Szczerba 2005; Munari et al. 2007), perhaps in a triple system with another tertiary B-type star (Chesneau et al. 2014b). Both V1309 Sco and V838 Mon exhibited double-peaked light curves, of shorter duration and brightness than those of SN Hunt 248 and NGC 4490-OT, but similar in multipeaked morphology. If they are mergers, the relatively long durations of SNHunt 248 and NGC 4490-OT, compared with V1309 Sco and V838 Mon, are to be expected from their relatively high progenitor masses. However, it is important to note that simulations of common-envelope outflows that are shock-energised by the binary's orbital energy input (e.g. Pejcha, Metzger \& Tomida 2016a; MacLeod et al. 2017) have not yet reproduced the high outflow velocities we have measured for SN Hunt $248\left(\sim 1200 \mathrm{~km} \mathrm{~s}^{-1}\right.$; Mauerhan et al. 2015), so the apparent trend in Fig. 12 has yet to be theoretically established at the high-mass end. More explosive forms of energy input that might result in fast $\sim 1000 \mathrm{~km} \mathrm{~s}^{-1}$ outflow velocities have been proposed to occur during the common-envelope evolution of massive stars (see Podsiadlowski et al. 2010; Soker \& Kashi 2013; Tsebrenko \& Soker 2013), but it is not clear if such effects would result in a continuation or deviation from the apparent trend in Fig. 12.

A multipeaked light curve might be a natural consequence of a stellar merger or common-envelope ejection. A close binary of evolved massive stars which are headed for a merger will experience mass transfer, and this can occur even if the primary radius does not fully fill its Roche lobe, but fills it up with material from a slow wind (e.g. wind Roche lobe overflow, WRLOF; Abate et al. 2013). RLOF may be non-conservative and WRLOF is non-conservative by nature (i.e. some mass is lost rather than exchanged). The process leads to the build-up of CSM with enhanced density in the equatorial plane of the binary, forming a spiral pattern that tightens with increasing radius and forms a dense torus-like structure surrounding the binary (Ohlmann et al. 2016 ; Pejcha, Metzger \& Tomida 2016a,b). The subsequent explosive outflow from a mergerburst will encounter this toroidal CSM distribution and generate radiation from the resulting interaction (multiple peaks in the light 
curve). Any interaction-induced dust formation will mirror the geometry of the pre-existing CSM. Relatedly, the circumstellar environment of the purported post-merger system V838 Mon exhibits an equatorial overdensity of dust several hundred au in extent (Chesneau et al. 2014b). Hydrodynamic simulations have also shown that equatorially enhanced dust formation should be expected in the aftermath of mergers (Pejcha, Metzger \& Tomida 2016a).

In comparing SNHunt 248 to stellar mergers, we should note that the B4-B5 spectral type we have estimated for the remnant would be much hotter than that of the immediate aftermath of the purported complete merger V838 Mon, which became the coolest supergiant ever observed with $T \approx 2000 \mathrm{~K}$ (L3 spectral type; Loebman et al. 2014). The cool source is presumably the inflated merger product, apparently contracting on a thermal time-scale (Chesneau et al. 2014b). The relatively hot spectral type of the remnant of SN Hunt 248 suggests that, if the eruption did indeed stem from a merging binary, then the individual stars might have avoided a complete merger while ejecting their common envelope. We speculate that the purported pseudophotosphere of the cool hypergiant was destroyed with the ejection of the inflated common envelope, revealing the stellar photosphere(s) of the hotter B4-B5 star(s) inside.

With regard to binary origin, it is possible that the light of the remnant is dominated by a companion to the eruptive source, or perhaps even a third tertiary companion to a progenitor binary system which may have merged. The latter idea, although very speculative at this point, is motivated by the discovery of a tertiary B-type companion in the V838 Mon system, which eventually became heavily reddened by expanding ejecta dust $\sim 5 \mathrm{yr}$ after the event (Wisniewski et al. 2008; Tylenda et al. 2011). This comparison warrants continued monitoring of SN Hunt 248 and NGC 4490-OT. Tertiary stars of triple systems could play an important role in the merger of the tighter pair, as has been suggested for $\eta$ Car (Portegies Zwart \& van den Heuval 2016).

\subsubsection{Peculiar core-collapse SN?}

Finally, we discuss the possibility that the 2014 eruption of SN Hunt 248 was a terminal explosion. This speculation is warranted, given renewed deliberation on the fates of transients previously classified as non-terminal SN impostors, including the prototype SN impostor SN 1997bs, and SN2008S (Adams \& Kochanek 2015; Adams et al. 2016). Like SN Hunt 248, SN 1997bs exhibited relatively narrow spectral lines (no obvious sign of highvelocity ejecta), peaked at a luminosity below that of typical corecollapse $\mathrm{SNe}$, and had a much shorter duration than common $\mathrm{SNe}$ II-P. Interestingly, at $\sim 1 \mathrm{yr}$ post-eruption, SN 1997bs exhibited an optical remnant very similar in brightness to that of SN Hunt 248 (see Fig. 4), but which continued to fade during subsequent coverage (Kochanek, Szczygiel, \& Stanek 2012b). Remarkably, the most recent optical-IR data on SN 1997bs appear to be consistent with a terminal explosion, as few plausible combinations of obscuring dust and surviving stellar luminosity can explain the late-time data. Could SN Hunt 248 have been a terminal event, similar to what has been suggested for SN 1997bs? If so, then the UV-optical remnant could either be a companion star, or it could be residual SN emission that coincidentally appears similar to the attenuated B4-B5 supergiant SED we constructed. In the latter possibility, we might expect the light curve of the optical remnant to continue evolving similarly to SN 1997bs (see Fig. 4), underscoring the need for continued UV-IR observations.

\section{SUMMARY AND CONCLUDING REMARKS}

We have presented space-based observations of the aftermath of SN Hunt 248 with HST and Spitzer. The UV-optical SED is consistent with a B4-B5 supergiant attenuated by grey circumstellar extinction. Our modelling of the Spitzer data suggests that the dust responsible for the IR emission is composed of relatively large grains $(a \gtrsim 0.3 \mu \mathrm{m})$, has a mass of $\sim 10^{-6}-10^{-5} \mathrm{M}_{\odot}$ (depending on whether it is graphitic or silicate) and a temperature of $T_{\mathrm{d}} \approx$ $900 \mathrm{~K}$. The large grain size indicated by our modelling results is consistent with the grey extinction we infer for the UV-optical remnant. However, the extinction expected from the hot-dust component alone is significantly below the amount suggested by the best-matching UV-optical SED, prompting us to speculate on the presence of cooler dust not detected by our 3.6 and $4.5 \mu \mathrm{m}$ photometry. Future mid-IR observations with the James Webb Space Telescope (JWST) could reveal such cooler dust.

We revised our analysis of the precursor-star photometry, and showed that the SED is well matched by an F-type supergiant that also suffers grey circumstellar extinction but of lesser magnitude than the remnant. Comparison of the extinction-corrected photometry to rotating stellar models indicates that the initial mass of the star could be nearly $\sim 60 \mathrm{M}_{\odot}$, approximately twice the value estimated by Mauerhan et al. (2015).

We interpreted the 2014 outburst of SN Hunt 248 in the context of binary mergers, as in the very similar case of NGC 4490-OT (Smith et al. 2016b). If such an interpretation is correct, then the hot B4-B5 spectral type of the byproduct might suggest that the binary avoided a complete merger during the ejection of the common envelope. In this interpretation, it could be that the ejection of the common envelope resulted in the destruction of the cool hypergiant pseudophotosphere suggested by Mauerhan et al. (2015), and prompted the star's transition to B4-B5 spectral type. This hypothesis, of course, requires that the remnant light, particularly the UV flux, is dominated by the eruptive star, and not a binary companion or unrelated neighbouring source.

The nature of the stellar aftermath and the 2014 eruption will be elucidated further with future UV through IR monitoring of the source using HST and JWST. Specifically, additional observations to track the evolution of the SED will allow for the construction of more complex models involving a surviving central source(s) attenuated by an evolving dust component. If dust has continued to condense in the ejecta since the last observations, then we expect that the UV-optical extinction will increase, regardless of whether the central light is from the eruptive source or a binary companion that has also been engulfed by the ejecta. If dust formation has ceased, however, then we might observe the future restrengthening of the optical flux, as the optical depth of an expanding dusty ejecta should decrease with geometric expansion over time as $\tau \propto t^{-2}$ (Kochanek et al. 2012b). In both cases, if dust is in a continually expanding unbound outflow, then it will also have cooled, and the 1-5 $\mu \mathrm{m}$ IR excess will fade as the flux shifts to longer wavelengths. On the other hand, if future observations reveal that the dust has remained hot and emitting at near-IR wavelengths, it would indicate that there could be an additional circumstellar dust component close to the stellar source, perhaps similar to the case of $\eta$ Car (e.g. Smith 2010) or dusty Wolf-Rayet binaries (e.g. Williams et al. 2012).

\section{ACKNOWLEDGEMENTS}

This work is based in part on observations made with the NASA/ESA Hubble Space Telescope, obtained from the Data 
Archive at the Space Telescope Science Institute (STScI), which is operated by the Association of Universities for Research in Astronomy (AURA), Inc., under NASA contract NAS5-26555. This work is also based in part on observations and archival data obtained with the Spitzer Space Telescope, which is operated by the Jet Propulsion Laboratory, California Institute of Technology, under a contract with NASA; support was provided by NASA through an award issued by JPL/Caltech. AVF's supernova group is also supported by Gary \& Cynthia Bengier, the Richard \& Rhoda Goldman Fund, the Christopher R. Redlich Fund, the TABASGO Foundation and the Miller Institute for Basic Research in Science (U.C. Berkeley).

\section{REFERENCES}

Abate C., Pols O. R., Izzard R. G., Mohamed S. S., de Mink S. E., 2013, A\&A, 552, A26

Adams S. M., Kochanek C. S., 2015, MNRAS, 452, 2195

Adams S. M., Kochanek C. S., Prieto J. L., Dai X., Shappee B. J., Stanek K. Z., 2016, MNRAS, 460, 1645

Andriesse C. D., Donn B. D., Viotti R., 1978, MNRAS, 185, 771

Blagorodnova N. et al., 2017, ApJ, 834, 107

Bond H. E. et al., 2003, Nature, 422, 405

Burggraf B. et al., 2015, A\&A, 581, A12

Cardelli J. A., Clayton G. C., Mathis J. S., 1989, ApJ, 345, 245

Castelli F., Kurucz R. L., 2003, Proc. IAU Symp., 210, 20P

Chesneau O. et al., 2014a, A\&A, 563, A71

Chesneau O. et al., 2014b, A\&A, 569, L3

Davidson K. et al., 1999, AJ, 118, 1777

Dolphin A. E., 2000, PASP, 112, 1383

Ekström S. et al., 2012, A\&A, 537, A146

Fitzgerald M. P., 1970, A\&A, 4, 234

Fox O. D., Chevalier R. A., Dwek E., Skrutskie M. F., Sugerman B. E. K., Leisenring J. M., 2010, ApJ, 725, 1768

Fox O. D. et al., 2011, ApJ, 741, 7

Fox O. D. et al., 2015, MNRAS, 454, 4366

Gall C. et al., 2014, Nature, 511, 326

Harwit M., Malfait K., Decin L., Waelkens C., Feuchtgruber H., Melnick G. J., 2001, ApJ, 557, 844

Heger A., Fryer C. L., Woosley S. E., Langer N., Hartmann D. H., 2003, ApJ, 591, 288

Hildebrand R. H., 1983, QJRAS, 24, 267

Humphreys R. M., 1978, ApJS, 38, 309

Humphreys R. M., Davidson K., 1994, PASP, 106, 1025

Humphreys R. M., Strecker D. W., Ney E. P., 1971, ApJ, 167, L35

Humphreys R. M., Martin J. C., Gordon M. S., Jones T. J., 2016, ApJ, 826, 191

Jura M., Kleinmann S. G., 1990, ApJ, 351, 583

Justham S., Podsiadlowski P., Vink J. S., 2014, ApJ, 796, 121

Kankare E. et al., 2015, A\&A, 581, L4

Kashi A., Soker N., 2008, New Astron., 13, 569

Kashi A., Soker N., 2010, ApJ, 723, 602

Kochanek C. S., 2014, ApJ, 785, 28

Kochanek C. S., Beacom J. F., Kistler M. D., Prieto J. L., Stanek K. Z., Thompson T. A., Yüksel H., 2008, ApJ, 684, 1336

Kochanek C. S., Szczygiel D. M., Stanek K. Z., 2011, ApJ, 737, 76

Kochanek C. S., Khan R., Dai X., 2012a, ApJ, 759, 20

Kochanek C. S., Szczygieł D. M., Stanek K. Z., 2012b, ApJ, 758, 142

Kochanek C. S., Adams S. M., Belczynski K., 2014, MNRAS, 443, 1319

Lagadec E., Zijlstra A. A., Oudmaijer R. D., Verhoelst T., Cox N. L. J., Szczerba R., Mékarnia D., van Winckel H., 2011, A\&A, 534, L10

Langer N., 2012, AR\&A, 50, 107

Loebman S. R. et al., 2015, AJ, 149, 17

Lovegrove E., Woosley S. E., 2013, ApJ, 769, 109
MacLeod M., Macias P., Ramirez-Ruiz E., Grindlay J., Batta A., Montes G., 2017, ApJ, 835, 282

Martins F., Plez B., 2006, A\&A, 457, 637

Massey P., Plez B., Levesque E. M., Olsen K. A. G., Clayton G. C., Josselin E., 2005, ApJ, 634, 1286

Mauerhan J. C. et al., 2015, MNRAS, 447, 1922

Mulders G. D., Min M., Dominik C., Debes J. H., Schneider G., 2013, A\&A, 549, A112

Munari U. et al., 2007, A\&A, 474, 585

Muller S., Dinh-V-Trung, Lim J., Hirano N., Muthu C., Kwok S., 2007, ApJ, 656,1109

Ohlmann S. T., Röpke F. K., Pakmor R., Springel V., 2016, ApJ, 816, L9

Paczyński B., 1971, AR\&A, 9, 183

Pejcha O., Metzger B. D., Tomida K., 2016a, MNRAS, 455, 4351

Pejcha O., Metzger B. D., Tomida K., 2016b, MNRAS, 461, 2527

Podsiadlowski P., Ivanova N., Justham S., Rappaport S., 2010, MNRAS, 406, 840

Portegies Zwart S. F., van den Heuvel E. P. J., 2016, MNRAS, 456, 3401

Robinson G., Mitchell R. M., Aitken D. K., Briggs G. P., Roche P. F., 1987, MNRAS, 227, 535

Rodgers A. W., 1971, ApJ, 165, 665

Shenoy D. et al., 2016, AJ, 151, 51

Shiode J. H., Quataert E., 2014, ApJ, 780, 96

Smartt S. J., Eldridge J. J., Crockett R. M., Maund J. R., 2009, MNRAS, 395, 1409

Smith N., 2010, MNRAS, 402, 145

Smith N., 2014, ARA\&A, 52, 487

Smith N., Ferland G. J., 2007, ApJ, 655, 911

Smith N., Arnett W. D., 2014, ApJ, 785, 82

Smith N., Frew D. J., 2011, MNRAS, 415, 2009

Smith N., Tombleson R., 2015, MNRAS, 447, 598

Smith N., Morse J. A., Davidson K., Humphreys R. M., 2000, AJ, 120, 920

Smith N., Gehrz R. D., Hinz P. M., Hoffmann W. F., Hora J. L., Mamajek E. E., Meyer M. R., 2003, AJ, 125, 1458

Smith N., Vink J. S., de Koter A., 2004, ApJ, 615, 475

Smith N., Li W., Silverman J. M., Ganeshalingam M., Filippenko A. V., 2011, MNRAS, 415, 773

Smith N., Andrews J. E., Mauerhan J. C., Zheng W., Filippenko A. V., Graham M. L., Milne P., 2016a, MNRAS, 455, 3546

Smith N. et al., 2016b, MNRAS, 458, 950

Soker N., 2004, ApJ, 612, 1060

Soker N., Kashi A., 2013, ApJ, 764, L6

Sukhbold T., Ertl T., Woosley S. E., Brown J. M., Janka H.-T., 2016, ApJ, 821,38

Tsebrenko D., Soker N., 2013, ApJ, 777, L35

Tylenda R., Soker N., Szczerba R., 2005, A\&A, 441, 1099

Tylenda R. et al., 2011, A\&A, 528, A114

Van Dyk S. D., Matheson T., 2012a, in Davidson K., Humphreys R. M., eds, Eta Carinae and the Supernova Impostors, Vol. 384. Springer, New York, p. 249

Van Dyk S. D., Matheson T., 2012b, ApJ, 746, 179

Van Dyk S. D., Peng C. Y., King J. Y., Filippenko A. V., Treffers R. R., Li W., Richmond M. W., 2000, PASP, 112, 1532

Vanbeveren D., De Loore C., Van Rensbergen W., 1998, A\&AR, 9, 63

Vanbeveren D., Mennekens N., Van Rensbergen W., De Loore C., 2013, A\&A, 552, A105

Williams P. M., van der Hucht K. A., van Wyk F., Marang F., Whitelock P. A., Bouchet P., Setia Gunawan D. Y. A., 2012, MNRAS, 420, 2526

Wisniewski J. P., Clampin M., Bjorkman K. S., Barry R. K., 2008, ApJ, 683, L171

Woosley S. E., Heger A., 2012, ApJ, 752, 32

Yoon S.-C., Cantiello M., 2010, ApJ, 717, L62

Zorec J., Cidale L., Arias M. L., Frémat Y., Muratore M. F., Torres A. F., Martayan C., 2009, A\&A, 501, 297

This paper has been typeset from a $\mathrm{T}_{\mathrm{E}} \mathrm{X} / \mathrm{L} \mathrm{T} \mathrm{E} \mathrm{X}$ file prepared by the author. 